\title{
ARTICLE \\ Artesunate synergizes with sorafenib to induce ferroptosis in hepatocellular carcinoma
}

\author{
Zhong-jie Li ${ }^{1}$, Hui-qi Dai ${ }^{2}$, Xiao-wei Huang ${ }^{2}$, Ji Feng ${ }^{2}$, Jing-huan Deng ${ }^{3}$, Zi-xuan Wang ${ }^{2}$, Xiao-mei Yang ${ }^{1}$, Yu-jia Liu ${ }^{1}$, Yong Wu ${ }^{2}$,
} Pan-hong Chen ${ }^{1}$, Huan Shi ${ }^{1}$, Ji-gang Wang ${ }^{2,4,5}$, Jing Zhou ${ }^{1}$ and Guo-dong $\mathrm{Lu}^{1,2,3,6,7}$

Sorafenib is the first-line medication for advanced hepatocellular carcinoma (HCC), but it can only extend limited survival. It is imperative to find a combination strategy to increase sorafenib efficacy. Artesunate is such a preferred candidate, because artesunate is clinically well-tolerated and more importantly both drugs can induce ferroptosis through different mechanisms. In this study we investigated the combined effect of sorafenib and artesunate in inducing ferroptosis of HCC and elucidated the involved molecular mechanisms. We showed that artesunate greatly enhanced the anticancer effects of low dose of sorafenib against Huh7, SNU-449, and SNU-182 HCC cell lines in vitro and against Huh7 cell xenograft model in Balb/c nude mice. The combination index method confirmed that the combined effect of sorafenib and artesunate was synergistic. Compared with the treatment with artesunate or sorafenib alone, combined treatment induced significantly exacerbated lipid peroxidation and ferroptosis, which was blocked by $\mathrm{N}$-acetyl cysteine and ferroptosis inhibitors liproxstatin- 1 and deferoxamine mesylate, but not by inhibitors of other types of cell death (z-VAD, necrostatin-1 and belnacasan). In Huh7 cells, we demonstrated that the combined treatment induced oxidative stress and lysosome-mediated ferritinophagy, two essential aspects of ferroptosis. Sorafenib at low dose mainly caused oxidative stress through mitochondrial impairments and SLC7A11-invovled glutathione depletion. Artesunate-induced lysosome activation synergized with sorafenib-mediated pro-oxidative effects by promoting sequential reactions including lysosomal cathepsin B/L activation, ferritin degradation, lipid peroxidation, and consequent ferroptosis. Taken together, artesunate could be repurposed to sensitize sorafenib in HCC treatment. The combined treatment can be easily translated into clinical applications.

Keywords: Sorafenib; Artesunate; HCC; Ferroptosis; Mitochondria; Lysosome

Acta Pharmacologica Sinica (2021) 42:301-310; https://doi.org/10.1038/s41401-020-0478-3

\section{INTRODUCTION}

Hepatocellular carcinoma (HCC), which constitutes $90 \%$ of primary liver malignancies, is a deadly cancer. The 5-year survival rate of HCC is only $\sim 15 \%$ due to late diagnosis and impaired liver function [1]. HCC cells are commonly resistant to chemotherapeutic drugs [2]. There are two first-line systemic medications, namely, sorafenib and lenvatinib, available for advanced HCC [2]. These two targeted medications, along with another second-line drug, regorafenib, are chemical inhibitors of tumor angiogenesis involving multiple kinases (VEGFR, PDGFR, FGFR, etc.). However, the survival benefit of sorafenib is moderate, and only an extra 2-3 months of overall survival can be achieved based on two phase III multicenter randomized clinical trials [3]. The SHARP study demonstrated that sorafenib administration extends median overall survival from 7.9 to 10.7 months [3], while another study conducted in HBVdominant Asian HCC patients also revealed an elongation of median overall survival from 4.2 to 6.5 months [4]. Moreover, many HCC patients do not tolerate sorafenib toxicity, and some acquire adaptive resistance $[5,6]$. It is thus imperative to find an effective combination approach to sensitize HCC to systemic sorafenib treatment and improve its efficacy.

Because sorafenib is the standard of care for advanced HCC, multiple previous or ongoing studies have applied this combined strategy in HCC experimental animal models and/or in randomized clinical trials. Most studies have combined sorafenib with existing clinical (or preclinical) drugs with a known mechanism of action (MOA) [7]. For example, sorafenib has been combined with inhibitors of mammalian target of rapamycin (everolimus) [8, 9], tyrosine kinase (ibrutinib) [10], MEK (Cl-1040) [11], 26S proteasome (bortezomib) [12], etc. This strategy, however, requires precise patient selection based on molecular subclassification and biomarker availability. Because HCC is a highly heterogeneous disease, a single combination regimen may only benefit a small proportion of HCC patients. Furthermore, this approach is costly and potentially unaffordable. To date, only one recent phase III

\footnotetext{
${ }^{1}$ Department of Physiology, School of Preclinical Medicine, Guangxi Medical University, Nanning 530021, China; ${ }^{2}$ Department of Toxicology, School of Public Health, Guangxi Medical University, Nanning 530021, China; ${ }^{3}$ Guangxi Colleges and Universities Key Laboratory of Prevention and Control of Highly Prevalent Diseases, Guangxi Medical University, Nanning 530021, China; ${ }^{4}$ Artemisinin Research Center and Institute of Chinese Materia Medica, China Academy of Chinese Medical Sciences, Beijing 100700, China;

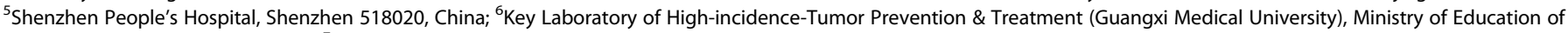
China, Nanning 530021, China and ${ }^{7}$ Cancer Science Institute of Singapore, National University of Singapore, 14 Medical Drive, Singapore 117599 , Singapore Correspondence: Ji-gang Wang (jgwang@icmm.ac.cn) or Jing Zhou (gardenia_zhou@hotmail.com) or Guo-dong Lu (golden_lu@hotmail.com)

These authors contributed equally: Zhong-jie Li, Hui-qi Dai, Xiao-wei Huang
}

Received: 19 March 2020 Accepted: 6 July 2020

Published online: 22 July 2020 
clinical trial combining sorafenib with the VEGFR2 inhibitor ramucirumab reached its primary endpoint of improving overall survival in HCC patients with high serum alpha-fetoprotein levels more than $400 \mathrm{ng} / \mathrm{mL}$ [13].

On the other hand, some other studies have aimed to increase sorafenib sensitivity by using well-tolerated nutrients or phytochemicals despite their lack of a clear MOA. This strategy, which is characterized by low toxicity and good affordability, may benefit a larger number of HCC patients. Vitamin K1 has been studied in combination with sorafenib in HCC and other solid tumors [14]. In addition, several phytochemicals, including fisetin [15], magnolol [16] and extracts from Antrodia cinnamomea [17], have been tested with sorafenib in preclinical studies. The synergistic effect of these agents involves enhanced apoptosis and impaired cell migration. It remains unclear whether these combinations are effective in clinical settings.

Ferroptosis is a newly identified form of iron-dependent programmed cell death that results from lethal lipid peroxidation $[18,19]$. Both iron chelators and lipophilic antioxidants can suppress ferroptosis. Ferroptosis sensitivity can be enhanced by excessive iron import through the interaction between transferrin and transferrin receptor (TFRC) or through increased free iron availability resulting from selective ferritin degradation (also referred to as ferritinophagy). In addition, impairments in phospholipid peroxidases, particularly glutathione peroxidase 4 and the upstream cystine transporter SLC7A11 (also called System Xc-), result in cell vulnerability to uncontrolled lipid peroxidation and ferroptosis. Thus, free iron accumulation and lipid peroxidation constitute two essential aspects of ferroptosis. The direct effect of sorafenib on HCC cells results from the induction of ferroptosis rather than apoptosis [20]. Sorafenib can directly inhibit SLC7A11 and consequently abolish glutathione biosynthesis [20]. As a result, depleted glutathione (GSH) cannot further protect cells from oxidative stress and lipid peroxidation. It is thus tempting to hypothesize that compounds that are capable of targeting other essential aspects of ferroptosis may synergize with sorafenib to inhibit HCC.

Artesunate is an ideal compound to test this hypothesis for the following two reasons. First, artesunate can induce ferroptosis independent of SLC7A11 [21, 22]. Our team [23] and others have reviewed how artesunate and artemisinin exert their antimalaria and non-antimalarial activities. We have convincingly shown that artemisinin has distinct targets in malaria parasites [24] and in cancer cells [25]. In malaria parasites, artemisinin's action primarily depends on heme biosynthesis. In contrast, artesunate preferably accumulates in lysosomes, activates lysosome function, and promotes ferritin degradation and free ferrous $\left(\mathrm{Fe}^{2+}\right)$ release in HeLa cervical cancer cells [25]. This artesunate-mediated effect on the lysosome and cellular iron accumulation may also occur in $\mathrm{HCC}$ cells, and artesunate may further synergize with sorafenib to induce ferroptosis. Second, as a semisynthetic derivative of the antimalarial magic drug artemisinin, water-soluble artesunate is well tolerated in clinical patients and relatively affordable [23]. Therefore, we hypothesize that artesunate is a preferred candidate to synergize with sorafenib to inhibit HCC. In the present study, we investigated the combined effect of sorafenib and artesunate in inducing ferroptosis in HCC cells in vitro and in xenograft tumors in vivo and determined the involved molecular mechanisms.

\section{MATERIALS AND METHODS}

\section{Cell culture and treatments}

The HepG2, SNU-182 and SNU-449 HCC cell lines were purchased from the American Type Culture Collection (Manassas, USA). Huh7 cell line was obtained from the Shanghai Institute of Biochemistry and Cell Biology (Shanghai, China). These cells were maintained in DMEM or RPMI-1640 medium containing $10 \%$ fetal calf serum from Gibco (Newcastle, Australia) in a humidified $5 \% \mathrm{CO}_{2}$ incubator at $37^{\circ} \mathrm{C}$.
Reagents and antibodies

Sorafenib was obtained from TargetMol (Shanghai, China); artesunate was obtained from Macklin (Shanghai, China); liproxstatin-1, ferrostatin-1, deferoxamine (DFO) mesylate, necrostatin-1 (Necro-1), SAR405, belnacasan, and z-VAD-FMK (z-VAD) were obtained from MedChemExpress (Shanghai, China); and N-acetyl cysteine (NAC), L-buthionine sulfoximine (BSO) and other chemicals were obtained from Sigma-Aldrich (Shanghai, China) unless otherwise indicated. Specific primary antibodies against FTL (ab69090) and FTH (ab75972), transferrin (ab82411) and TFRC (ab84036) were purchased from Abcam (Shanghai, China); and $a-$ tubulin antibody (\#T-5168) was purchased from Sigma-Aldrich.

Detection of cell viability, cell death, and colony formation Cell viability was assessed by the MTT assay (Beyotime, Shanghai, China). The effect of the drug combination was determined by the combination index $(\mathrm{Cl})$ method using CompuSyn software [26]. Cell death was determined by two different methods using a CytoFLEX flow cytometer (Beckman Coulter, Atlanta, USA) as described previously [27]. In the first method, subG1 (dead) cells were detected. The second method, the propidium iodide (PI) exclusion assay, captured cells with impaired cell membranes. For the colony formation assay, one thousand cells were seeded in 12-well plates and treated with the indicated drugs for 10 days. After crystal violet staining, colony images were analyzed with ImageJ software [28].

Determination of ROS levels and the mitochondrial membrane potential

CM- $\mathrm{H}_{2}$ DCFDA and MitoSOX Red (Thermo Fisher, Shanghai, China) were used for the detection of reactive oxygen species (ROS) and mitochondria-derived superoxide. Tetramethylrhodamine, methyl ester (TMRM, purchased from Thermo Fisher) was used to monitor the mitochondrial membrane potential. TMRM fluorescent signals were analyzed by both flow cytometry and an inverted EVOS $^{\mathrm{TM}}$ fluorescence microscope (Thermo Fisher).

Determination of lipid peroxidation

BODIPY $^{\text {TM }}$ 581/591 C11 (Thermo Fisher) was used to detect lipid peroxidation according to the manufacturer's instructions. The contents of GSH and malondialdehyde (MDA) were quantified by respective biochemical assay kits (BioVision, Milpitas, USA) with a SPARK $10 \mathrm{M}$ multimode microplate reader (Tecan, Morrisville, USA).

Measurement of ATP and the metabolic oxygen consumption rate ATP levels were quantified with an assay kit from Beyotime. A Seahorse live-cell metabolic assay (Agilent, Santa Clara, USA) was used to determine the mitochondrial oxygen consumption rate (OCR) as previously described [29]. Briefly, after 20,000 cells had been seeded in the plate overnight, the medium was replaced with XF medium. The cells were then assayed under basal conditions with or without drug treatments for $1 \mathrm{~h}$, subsequently with mitochondrial poisons according to the manufacturer's instructions.

Measurement of lysosomal activity and free $\mathrm{Fe}^{2+}$ ion levels Lysosomal enzymatic activity was detected with Magic Red Cathepsin B or Cathepsin L reagents (Bio-Rad, Shanghai, China). After being treated as indicated, the cells were reacted with the reagents for $30 \mathrm{~min}$ before fluorescence measurements. LysoTracker $^{\mathrm{TM}}$ Red (Thermo Fisher) was used to determine lysosomal acidity. The cellular content of free $\mathrm{Fe}^{2+}$ ions was determined by a biochemical kit obtained from Sigma-Aldrich as instructed by the manufacturer's manual.

Nude mouse xenograft study

The mouse experiment was approved by the Guangxi Medical University Institutional Animal Care and Use Committees and carried out in accordance with the Association for Assessment and Accreditation of Laboratory Animal Care guidelines. A total of 20 
male Balb/c nude mice aged 6-8 weeks were purchased from Hunan SJA Laboratory Animal Co., Ltd. (Changsha, China). Five million Huh7 cells were inoculated into the right flanks of the mice. When the tumor size reached $80-100 \mathrm{~mm}^{3}$, the mice were randomly divided into four groups and administered artesunate $(30 \mathrm{mg} / \mathrm{kg}$ mouse weight) alone, sorafenib $(20 \mathrm{mg} / \mathrm{kg}$ mouse weight) alone, a combination of artesunate and sorafenib, or the same volume of PBS by gavage every other day. The size of the tumor nodules was measured with a digital caliper every day using the formula (length $\times$ width $\left.^{2}\right) / 2$.

Immunohistochemical (IHC) staining and Western blotting IHC staining was conducted as previously described [29]. Briefly, tumor tissue sections were incubated with specific primary antibodies overnight at $4{ }^{\circ} \mathrm{C}$ before detection with a standard Biotin-Streptavidin HRP detection system (Zsbio, Beijing, China). The tissue sections were also stained according to a standard hematoxylin and eosin (H\&E) staining protocol. Western blotting was conducted by enhanced chemiluminescence [29].

Statistical analyses

All statistical analyses were performed using SPSS 21.0 software (IBM SPSS Statistics for Windows). ANOVA followed by LSD and two-sided Student's $t$ test were applied to analyze the experimental differences. A $P$ value $<0.05$ was regarded as statistically significant.

\section{RESULTS}

Artesunate sensitized HCC cells to sorafenib to induce death of HCC cells in vitro

We first examined whether sorafenib and artesunate act synergistically in HCC cells. Artesunate alone inhibited the proliferation of HepG2 cells. When combined with increasing doses of sorafenib, artesunate further decreased cell proliferation (Fig. 1a). The synergistic effect was more evident in Huh7, SNU449 , and SNU-182 cells, even when sorafenib was administered at doses as low as $0.5-2 \mu \mathrm{M}$ (Fig. 1a). The combination index method confirmed that the combined effect of sorafenib and artesunate was synergistic (Supplementary Fig. 1a). Thus, in the following experiments, we applied the combination of $25 \mu \mathrm{M}$ artesunate and $2 \mu \mathrm{M}$ sorafenib to study the molecular mechanisms involved in their synergistic effect.

Cell survival analysis demonstrated that the combined treatment caused extensive cell death. More importantly, this cell death was blocked by pretreatment with NAC (an ROS scavenger) or SAR405 (a PIK3C3/Nps34 inhibitor that prevents autophagy) (Fig. 1b) but not inhibitors of different types of cell death, namely, z-VAD (a pan-caspase inhibitor that prevents apoptosis), Necro-1 (a RIP kinase inhibitor that prevents necroptosis), and belnacasan (a caspase-1 inhibitor that prevents inflammation-mediated pyroptosis). These results suggest the possible involvement of oxidative stress and autophagy in the induction of cell death. The colony formation assay further confirmed that the combined treatment markedly decreased colony growth after 10 days of treatment (Fig. 1c). Taken together, the above results indicated that artesunate and sorafenib had synergistic effects on ROSdependent cell death.

The combined treatment inhibited xenograft tumors in vivo through extensive cell death

Next, we explored whether combined treatment with sorafenib and artesunate can inhibit HCC tumor growth in vivo. The Balb/c nude mice with ectopic xenograft HCC were given artesunate (30 mg/kg body weight), sorafenib $(20 \mathrm{mg} / \mathrm{kg})$, combined treatment or the same volume of PBS (as control) by gavage every other day. The combined treatment did not have observable toxic effects on the weight of the mice (Fig. 2a, left panel) but compared with PBS control and single treatment, significantly retarded tumor growth (Fig. 2a, right panel) and reduced the size of the tumor nodules (Fig. 2b). More importantly, H\&E pathological staining (Fig. 2c) revealed that the combined treatment caused extensive cell death, as evidenced by smeared cell morphology and the disappearance of nuclear staining. Although treatment with sorafenib alone or artesunate alone also caused cell death, the cell death areas in the sorafenib alone- and artesunate alonetreated groups were significantly smaller than that in the combined treatment group. The Ki-67 IHC staining results further suggested that in vivo tumor cell proliferation was significantly impaired by the combined treatment (Fig. 2d). Therefore, the results observed in the xenograft mice consistently demonstrated that sorafenib and artesunate had a synergistic effect in impairing HCC xenograft tumor growth and inducing cell death in vivo.

The combined treatment-induced lipid peroxidation and ferroptosis

By using the ferroptosis inhibitors liproxstatin-1 (Lipro) and DFO, we confirmed that the induced cell death by the combined treatment resulted from ferroptosis (Fig. 3a). Lipro neutralizes lipid peroxidation, while DFO chelates iron; both of these processes are essential aspects of ferroptosis. The combined treatment consistently caused lipid peroxidation, as assessed by BODIPY C11 (Fig. $3 \mathrm{~b}$ ) and MDA production (one of the final products of polyunsaturated fatty acid peroxidation, Fig. 3c). NAC and Lipro, however, prevented the induction of lipid peroxidation (Fig. 3b). It is noteworthy that artesunate alone had a stronger stimulatory effect than sorafenib alone on the induction of lipid peroxidation (Fig. 3b, c), suggesting that artesunate may act mainly through modulation of lipid peroxidation. In contrast, GSH was diminished by the combined treatment and sorafenib alone but not artesunate alone (Fig. 3d). This sorafenib-mediated GSH reduction is consistent with the previous finding that sorafenib can block SLC7A11 function and subsequent GSH synthesis [20].

Artesunate alone was previously reported to induce ferroptosis $[21,22]$. We thus used higher doses to replicate these results. Artesunate at concentrations as high as $200 \mu \mathrm{M}$ reduced HCC cell viability, and the effect was reversed by pretreatment with the ferroptosis inhibitors Lipro, DFO and ferrostatin-1 but not z-VAD or Necro-1 (Supplementary Fig. 1b). These results suggested that a high dose of artesunate induced ferroptotic cell death. Consistently, a high dose of artesunate $(200 \mu \mathrm{M})$ increased lipid peroxidation, which was reversed by Lipro and ferrostatin-1 pretreatment (Supplementary Fig. 1c). Taken together, the above results showed that the combined treatment-induced lipid peroxidation and ferroptosis.

The combined treatment and sorafenib alone impaired mitochondrial functions

We further investigated the possible role of mitochondria in the induction of ROS-dependent cell death. The DCFDA assay results (Fig. 4a) showed that the combined treatment, but not sorafenib alone or artesunate alone, augmented cellular ROS production. In addition, the combined treatment increased the generation of mitochondrial-derived ROS (MDR; as assessed by MitoSOX Red in Fig. 4b). Notably, this effect was mainly elicited by sorafenib, although artesunate alone also induced a moderate increase in MDR levels. Consistently, sorafenib alone and the combined treatment caused a reduction in the mitochondrial membrane potential, as shown by decreased TMRM signaling (Fig. 4c). The essential role of ROS was further validated by NAC. NAC pretreatment abolished combined treatment-induced ROS (Fig. 3a) and MDR generation (Fig. 4b).

Mitochondria are the major subcellular organelles responsible for energy production. As expected, the combined treatment significantly repressed ATP production (Fig. 4d). Furthermore, NAC rescued ATP production (Fig. 4d). The Seahorse metabolic assay 
a

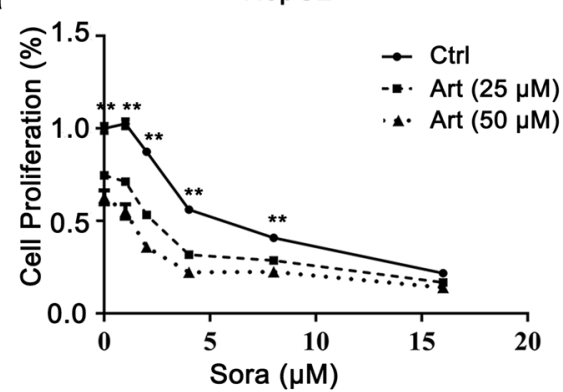

SNU182

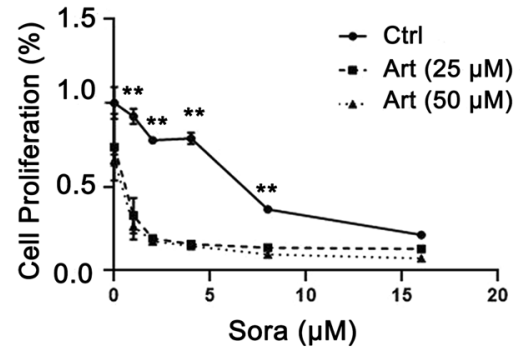

b

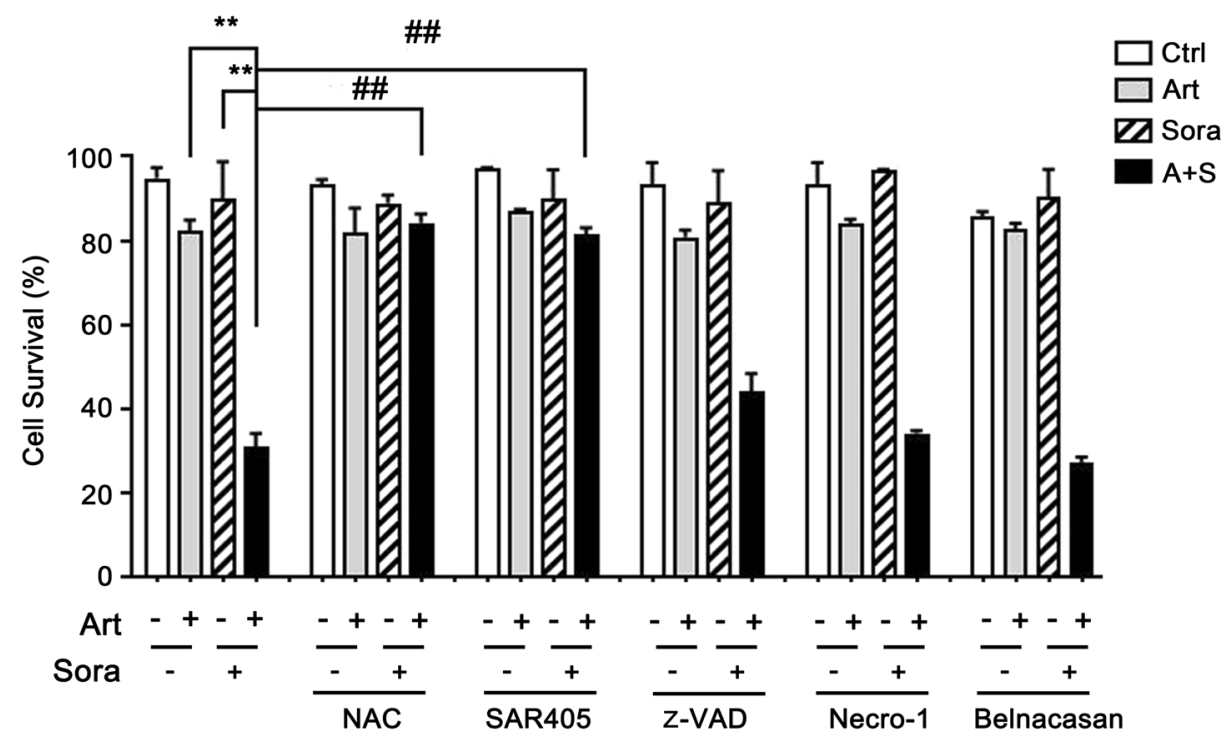

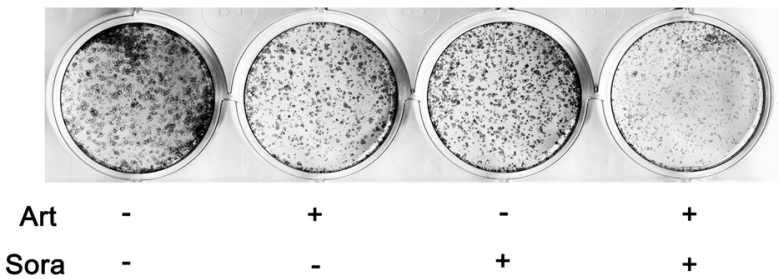

Huh7

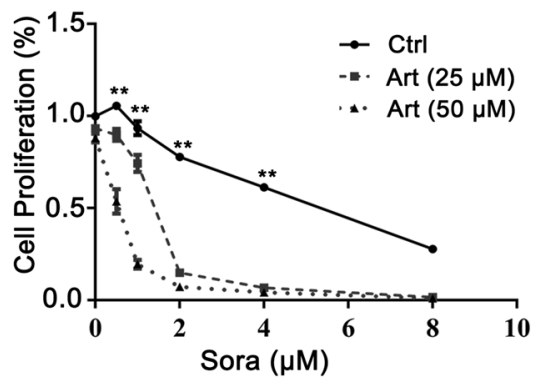

SNU449

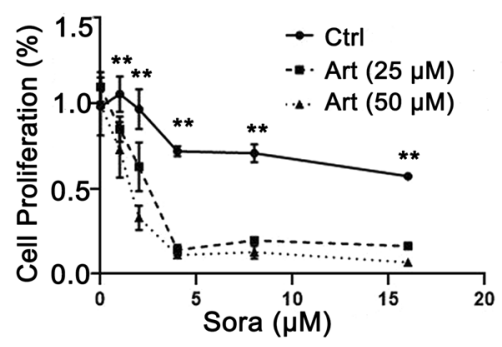

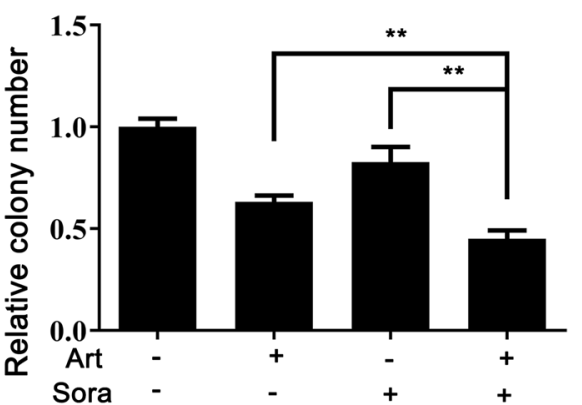

Fig. 1 Artesunate sensitized HCC cells to sorafenib-induced death. a HepG2, Huh7 and SNU-449 cells were treated with artesunate (Art) and/or sorafenib (Sora) as indicated for $24 \mathrm{~h}$, while SNU-182 cells were administered the same treatments for $12 \mathrm{~h}$. Cell proliferation was measured by the MTT assay. b Huh7 cells were treated with Art $(25 \mu \mathrm{M})$ and/or Sora $(2 \mu \mathrm{M})$ in the presence or absence of pretreatment with different inhibitors for $24 \mathrm{~h}$. These inhibitors included NAC (an ROS scavenger, $5 \mathrm{mM}$ ), SAR405 (an inhibitor of PIK3C3/Vps34 and consequent autophagy, $1 \mu \mathrm{M}$ ), z-VAD (a pan-caspase inhibitor, $40 \mu \mathrm{M}$ ), necrostatin-1 (an inhibitor of RIP kinase and consequent necroptosis, $20 \mu \mathrm{M}$ ), and belnacasan (an inhibitor of caspase-1 and consequent pyroptosis, $20 \mu \mathrm{M}$ ). The cell survival rate was determined by the PI exclusion assay. c Huh7 cells were seeded in 12-well plates and treated with Art $(10 \mu \mathrm{M})$ and Sora $(1 \mu \mathrm{M})$ for 10 days. A + S, the combination of Art and Sora; ${ }^{* *} P<0.01$ compared with Art alone or Sora alone; ${ }^{\# \#} P<0.01$ compared with the combination of Art and Sora. 
a

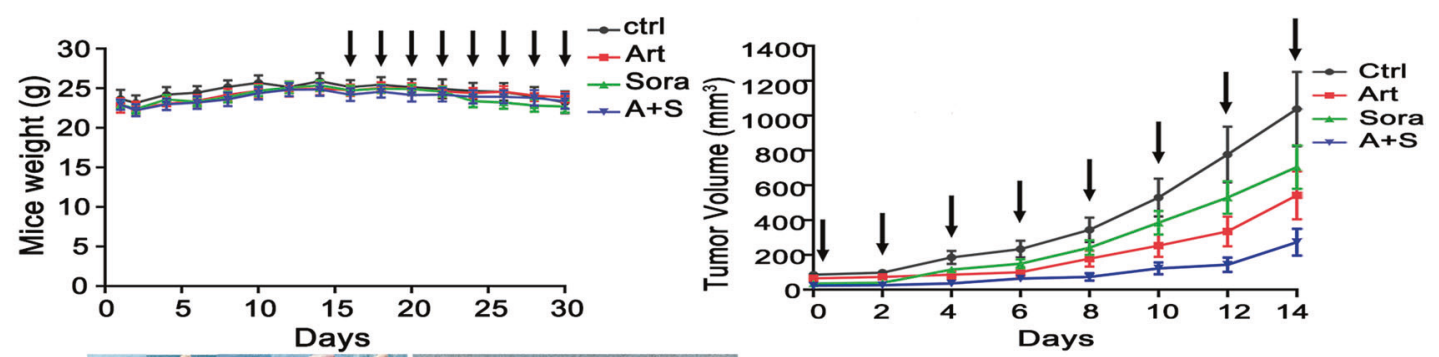

b

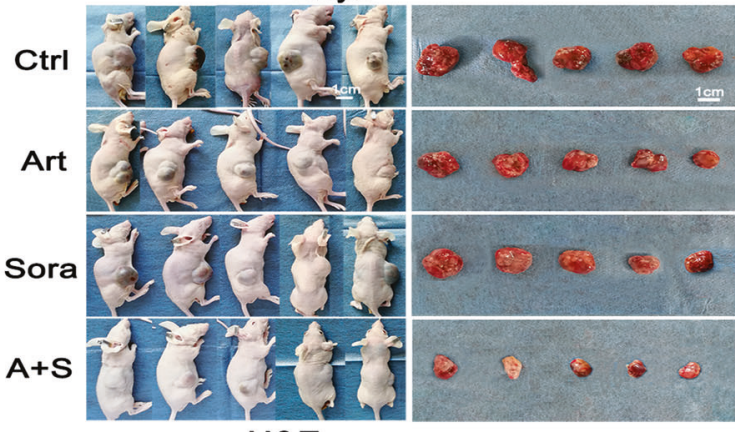

C
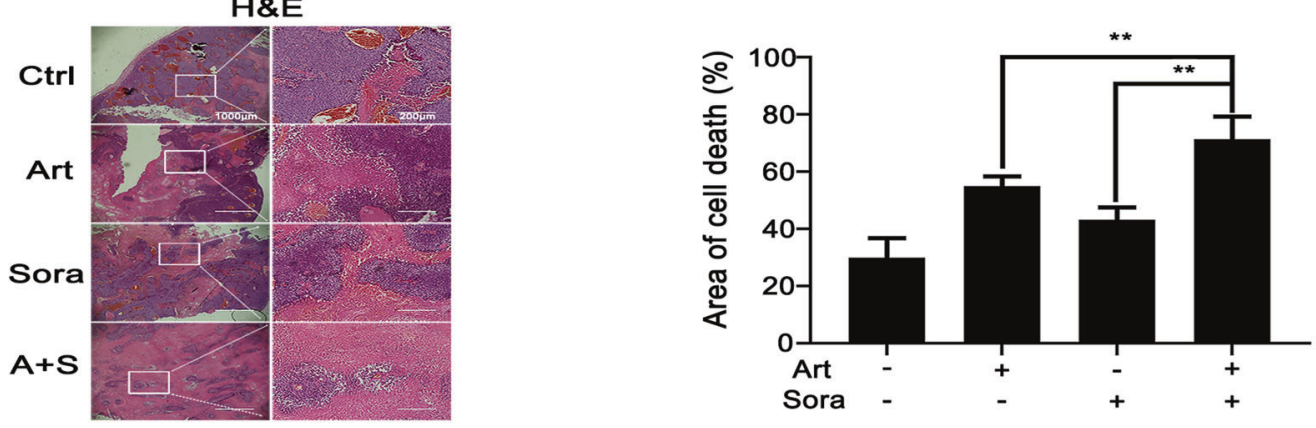

d
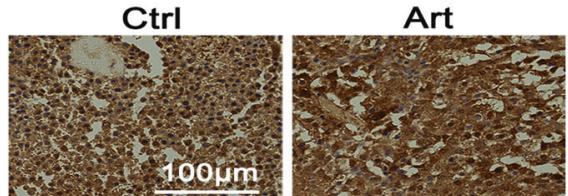

Ki67

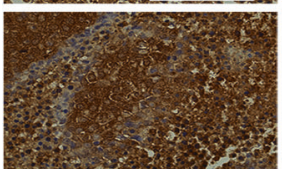

Sora

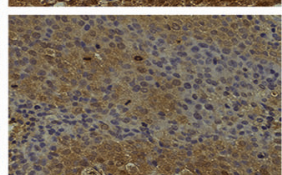

$A+S$

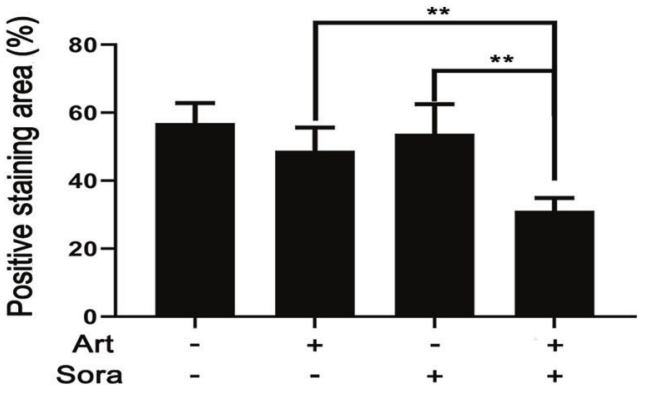

Fig. 2 The combined treatment inhibited xenograft tumors in vivo through extensive cell death. a Once the size of the inoculated xenograft nodules reached $80-100 \mathrm{~mm}^{3}$, the mice were randomly divided into four groups ( $n=5$ per group) and treated by gavage with Art (30 mg/kg body weight), Sora ( $20 \mathrm{mg} / \mathrm{kg}$ ), the combination of Art and Sora or PBS (control) every other day. The weight of the mice (left panel) and tumor growth (right panel) were monitored regularly. The arrows indicate drug treatment. $\mathbf{b}$ The mice were sacrificed, and the tumors were resected for subsequent experiments. c Xenograft tumor sections were stained with H\&E. The area of cell death was indicated by smeared cell morphology and faint nuclear staining. The area of cell death is presented in the right panel. $\mathbf{d}$ Tumor cell proliferation status was analyzed by $\mathrm{IHC}$ staining for Ki-67. ${ }^{* *} P<0.01$.

confirmed that the combined treatment reduced the ATP-linked respiratory OCR (Fig. 4e). The combined treatment caused protons to leak out of mitochondria, which was in line with the increases in MDR and ROS levels (Fig. 4a, b). Thus, these results demonstrated that the combined treatment impaired mitochondrial functions, particularly mitochondrial superoxide homeostasis and ATP generation.

The combined treatment increased lysosomal function and ferritin degradation

Because of the dependency of ferroptotic cell death induction on iron (Fig. 3a), we further investigated how iron metabolism is affected by the combined treatment. Ferritin and transferrin are two key iron-binding and iron-regulatory proteins in the body. The former stores iron within the cytoplasm, while the latter transports iron in the blood or body fluids. The results shown in Fig. 5a reveal that the combined treatment inhibited the expression of both ferritin light chain (FTL) and heavy chain (FTH). TFRC expression was decreased by the combined treatment as well. Bafilomycin A1 is a lysosomal inhibitor. Bafilomycin A1 pretreatment, however, markedly blocked FTL reduction, suggesting that FTL was degraded by the lysosome. Notably, bafilomycin A1 pretreatment also protected against induced cell death (Fig. 5b), further confirmed the essential role of the lysosome in the induction of ferroptosis. This result is 


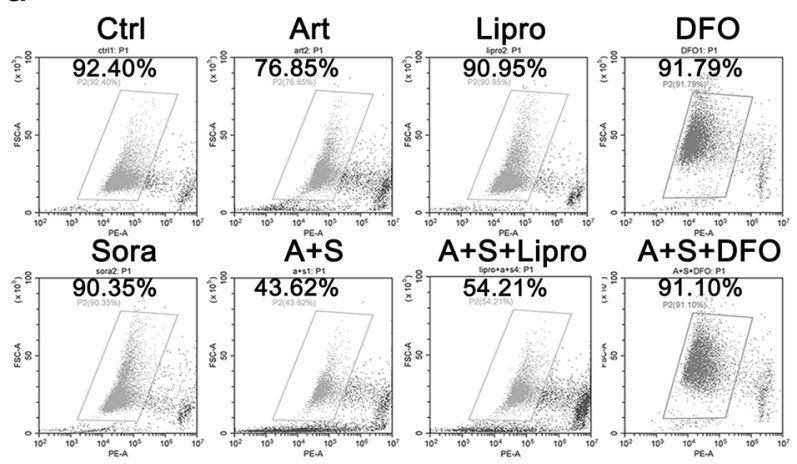

b

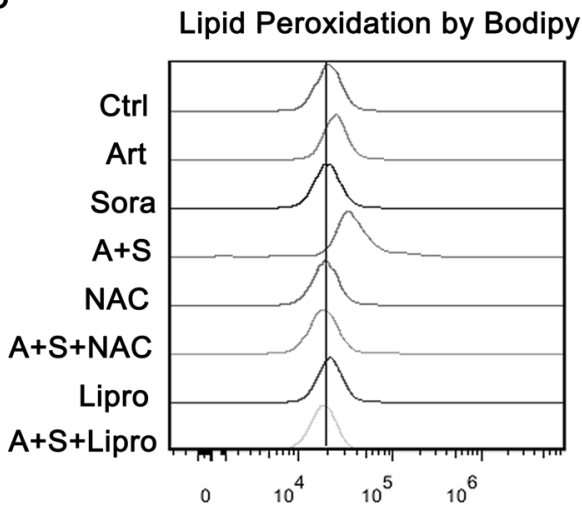

d

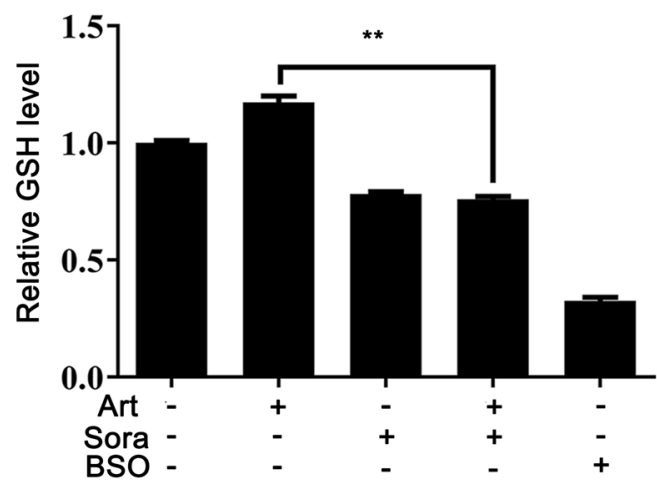

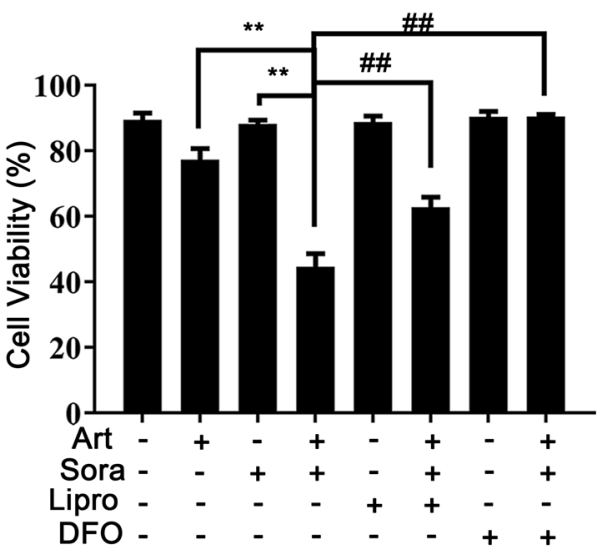

C
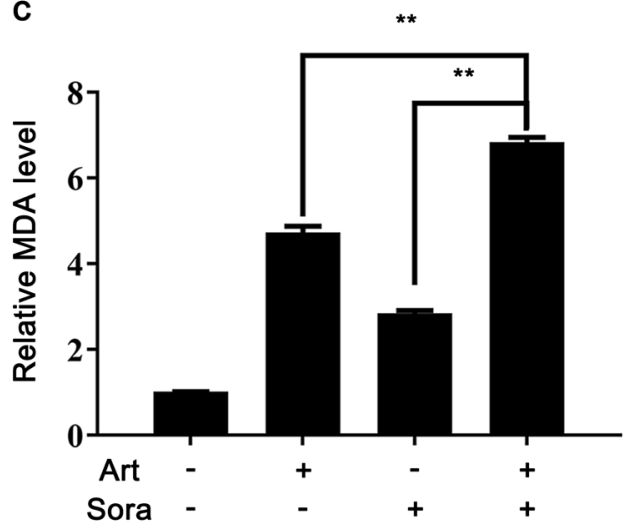

Fig. 3 The combined treatment-induced lipid peroxidation and ferroptosis. a Huh7 cells were treated as indicated with or without Liproxstatin-1 (Lipro, $10 \mu \mathrm{M})$ and deferoxamine (DFO, $100 \mu \mathrm{M})$. The cell survival rate was quantified by the PI exclusion assay. $\mathbf{b}$ Huh7 cells were treated as indicated with or without Lipro or NAC. The level of lipid peroxidation was determined with BODIPY 581/591 C11. After cell treatments, MDA production (c) and GSH content (d) were measured with respective assay kits as described. BSO (10 $\mu$ M) was used as a positive control. ${ }^{* *} P<0.01$.

consistent with the cell-protective effect of pretreatment with SAR405 (another autophagy inhibitor, Fig. 1b). Examination of lysosomal activity with LysoTracker Red dye revealed that the combined treatment and artesunate alone caused lysosomal activation (Fig. 5c). Moreover, lysosomal activation was further evidenced by increases in lysosomal cathepsin $L$ and cathepsin B activities, while bafilomycin A1 consistently reversed the activation of both enzymes (Fig. 5d, e). Taken together, these results suggested that lysosomal activation and ferritin degradation were essential for combined treatment-induced ferroptosis.

Finally, we determined the in vivo effects of the combined treatment on FTL degradation, free $\mathrm{Fe}^{2+}$ contents and lipid peroxidation. Similar to what was observed in the in vitro cell experiments (Fig. 5a), FTL protein expression in resected tumors was significantly repressed by the combined treatment (Fig. 6a). Consistently, the concentrations of free $\mathrm{Fe}^{2+}$ ions were increased in tumor nodules after combined treatment (Fig. 6b). In contrast, the MDA content in the tumor nodules (Fig. 6c) was increased by artesunate alone and increased more significantly by the combined treatment, which was in line with the results observed in cells in vitro (Fig. 3c).

\section{DISCUSSION}

The present study found that sorafenib and artesunate act synergistically to inhibit HCC through ferroptosis induction. Sorafenib 
a

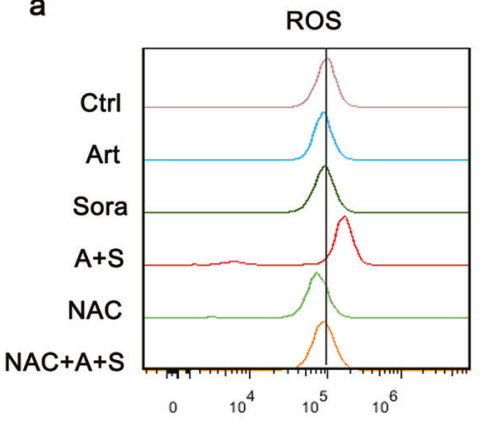

c

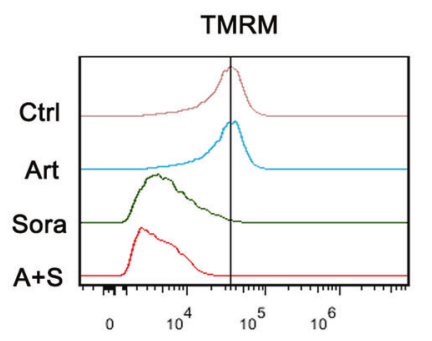

d

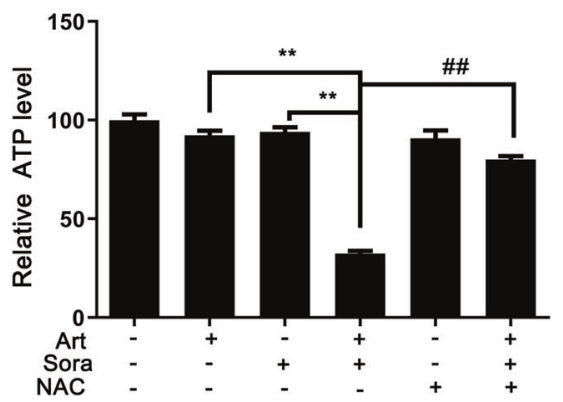

b
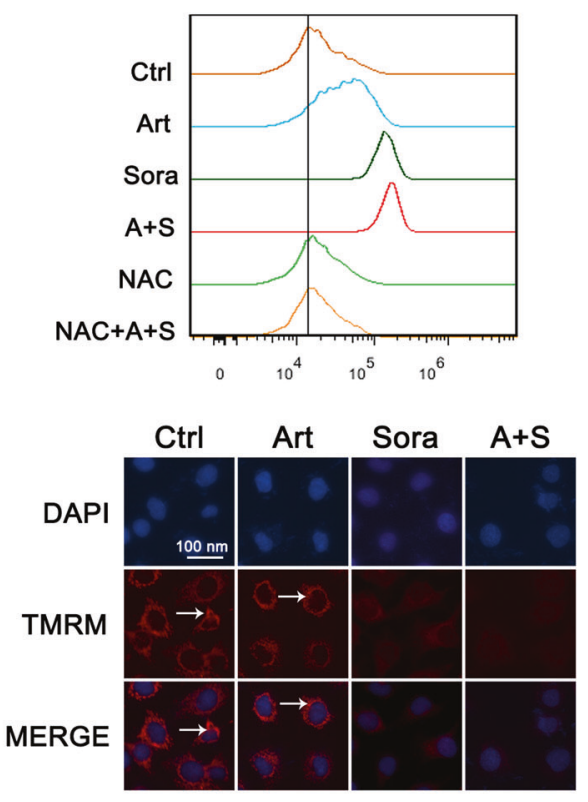

e

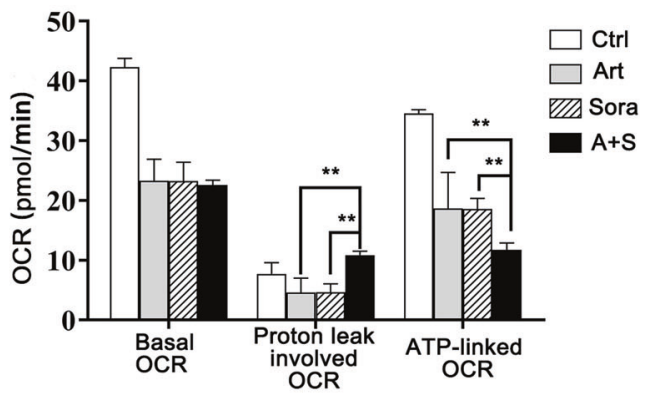

Fig. 4 The combined treatment impaired mitochondrial functions. Huh7 cells were treated as indicated in Fig. $3 \mathrm{~b}$. Then, the levels of ROS (a) and mitochondrial-derived ROS (b) were measured with DCFDA and MitoSOX Red, respectively. c The mitochondrial membrane potential was determined with TMRM by using flow cytometry (left panel) and fluorescence microscopy (right panel). d The cellular ATP levels were determined by biochemical assays after the indicated treatments. e Huh7 cells were washed and recultured in fresh XF medium with the indicated drugs for $1 \mathrm{~h}$. Then, the mitochondrial respiratory OCR was determined by Seahorse metabolic assay. Basal respiration OCR, proton leak-involved OCR and ATP-linked OCR are summarized. OCR oxygen consumption rate. ${ }^{* *} P<0.01$.

mainly promoted oxidative stress via impairment of mitochondrial function and blockage of SLC7A11-dependent GSH synthesis. Artesunate acted primarily on lysosomes and synergized with sorafenib to induce cathepsin $B / L$ activation and ferritin degradation. These two processes constitute the two essential aspects of ferroptosis in HCC cells in vitro and in xenograft tumors in vivo. The cartoon in Fig. $6 \mathrm{~d}$ depicts the signaling pathways through which the combined treatment sequentially induces ferroptosis.

Both sorafenib and artesunate are clinically available, so their combined application is feasible in clinical settings. Both drugs have the same MOA in inducing ferroptosis but act through different molecular mechanisms $[20,21]$. These common properties may enable them to be used in combination for HCC treatment. On the one hand, sorafenib mainly acted on oxidative stress. Even at concentrations as low as $2 \mu \mathrm{M}$, sorafenib alone effectively disrupted the mitochondrial membrane potential (Fig. 4c) and induced mitochondria-derived ROS generation (Fig. 4b). Furthermore, sorafenib alone decreased GSH content (Fig. 3d), as reported previously [20]. It has been observed that artesunate can also dose-dependently decrease GSH levels in head and neck cancer cells and ovarian cancer cells [30, 31]. However, in the present study, artesunate alone at a low dose had minimal effects on GSH content (Fig. 3d). This inconsistency may have been due to tissue specificity. Roh et al. reported that artesunate can activate the NRF2 antioxidant response element pathway and induce resistance to ferroptosis [30].

On the other hand, artesunate alone primarily increased lysosomal activity (Fig. 5c) and lipid peroxidation (Fig. 3b, c). The combined treatment further promoted lysosomal cathepsin B/L activity (Fig. 5d, e) and lysosome-dependent FTL degradation (Fig. 5a). This result, except for the difference in the dose of artesunate, was also observed in our previous report conducted in HeLa cells [25]. As a result of ferritin degradation, the cellular free iron pool may be increased and become available for the Fenton reaction and lipid peroxidation. Artesunate-induced lysosomal activation and sorafenib-induced oxidative stress thus constitute the two essential aspects of ferroptosis.

We also observed that the combined treatment downregulated transferrin receptor expression (Fig. 5a). TFRC helps to internalize transferrin-transported iron. TFRC has been found to be essential for the cytotoxicity of artemisinin-transferrin conjugates in prostate cancer cells [32]. In renal cell carcinoma cells, artesunate can increase TFRC expression [33]. However, in the present study, the combined treatment, but not artesunate alone, decreased 


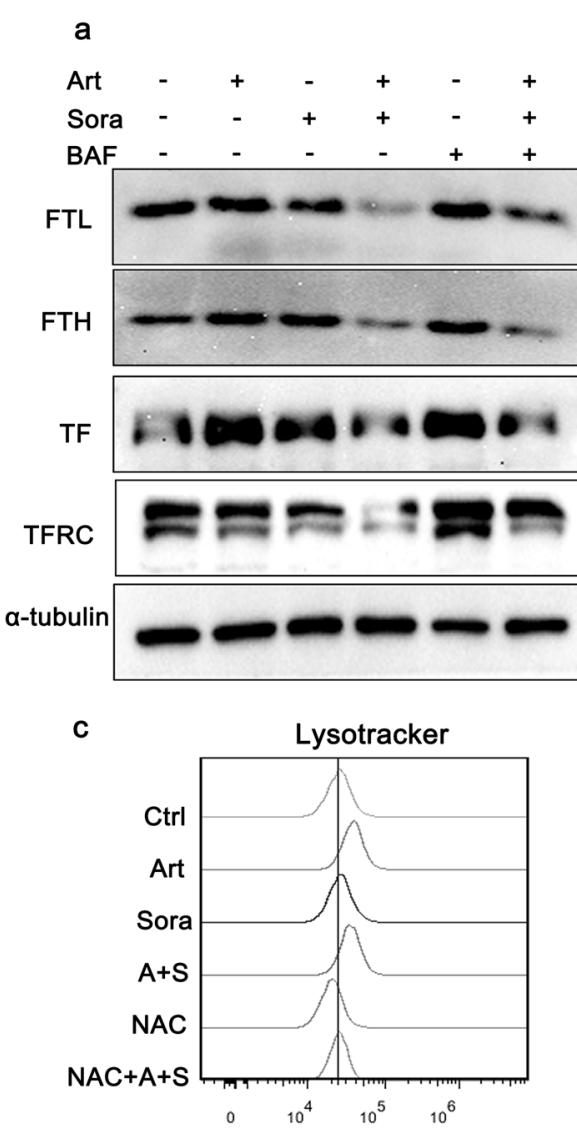

b
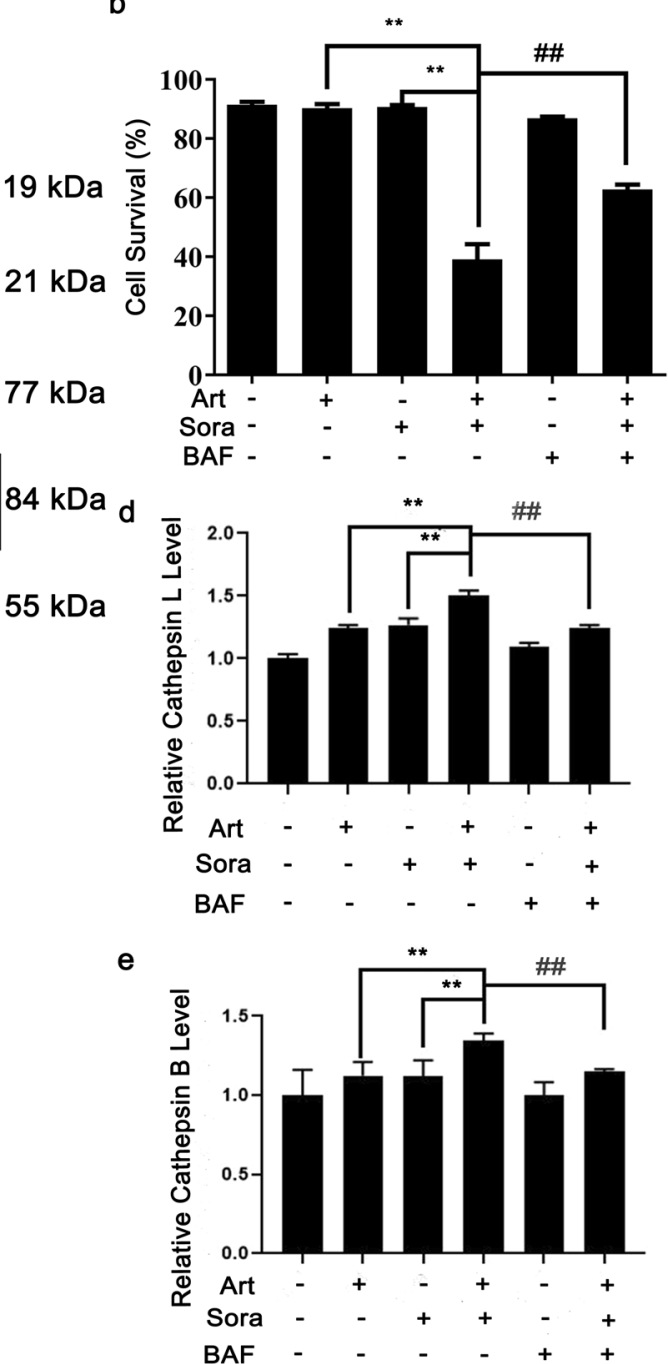

Fig. 5 The combined treatment-induced lysosomal activation and ferritin degradation. a Huh7 cells were treated as indicated with or without bafilomycin A1 (100 nM) for $12 \mathrm{~h}$ before Western blotting. b Huh7 cells were treated as indicated in (a) for $24 \mathrm{~h}$ before the cell survival rate was quantified. c Huh7 cells were treated as indicated in Fig. 4a before lysosomal activity analysis using LysoTracker Red. d, e Huh7 cells were treated as indicated with or without bafilomycin $A 1(100 \mathrm{nM})$ pretreatment for $12 \mathrm{~h}$. Then, the lysosomal enzyme activities of cathepsin $\mathrm{L}$ (d) and cathepsin B (e) were quantified. ${ }^{* *} P<0.01 .{ }^{\# \#} P<0.01$.

TFRC expression. This decrease was also accompanied by the degradation of FTL and FTH (Fig. 5a). In addition, bafilomycin A1 blocked TFRC protein degradation, suggesting that TFRC degradation was also dependent on lysosomal activation. We thus speculate that the decrease in TFRC may have resulted from a negative feedback of the increased free iron pool after FTL and FTH degradation. However, this warrants careful examination in future studies.

Notably, the synergistic effect of artesunate and sorafenib was studied previously. Vandewynckel et al. [34] reported that artesunate might attenuate tumor neovascularization and synergize with sorafenib to inhibit HCC cells in vitro and diethylnitrosamine-induced HCC in vivo in mice. Consistently, artesunate acts synergistically with sorafenib to inhibit metastatic renal cell carcinoma through the same mechanism [33]. A recent report also showed that artesunate sensitizes HCC cells to sorafenib to induce apoptosis through impairment of the PI3K/ AKT/mTOR pathway [35]. In the present study, combined treatment with artesunate and sorafenib caused extensive cell death in xenograft nodules in vivo (Fig. 2). It seems that this phenomenon could also be explained by impaired neoangiogenesis and consequent hypoxia and nutrient deprivation-induced cell death. However, our in vitro cell death experiments were carried out in the presence of sufficient oxygen and nutrients, suggesting that the direct action of the combined treatment was sufficient to induce cell death. Furthermore, we observed that the combined treatment induced consistent FTL degradation and accumulation of free $\mathrm{Fe}^{2+}$ ions and MDA (typical changes associated with ferroptosis) both in vitro and in vivo (Figs. 5 and 6). Thus, the induction of cell death in the xenograft nodules may have, at least partially, resulted from ferroptosis. More importantly, the experiments involving pretreatment with different cell death inhibitors (Fig. 1b) convincingly demonstrated that the combined treatment caused ferroptosis rather than apoptosis, necroptosis, or pyroptosis in HCC (Fig. 1). This conclusion is further supported by other findings that ferroptosis is a direct result of sorafenib alone [20].

The present findings might have important clinical indications. First, personalized HCC treatment with sorafenib may be feasible. Detection of the basal levels or expression changes in ferroptosis-dependent proteins via HCC biopsy or resected samples could help identify HCC patients who are sensitive or resistant to sorafenib. Several research groups including our own reported that the basal level of $\operatorname{ACSL} 4[36,37]$ predicts 
a

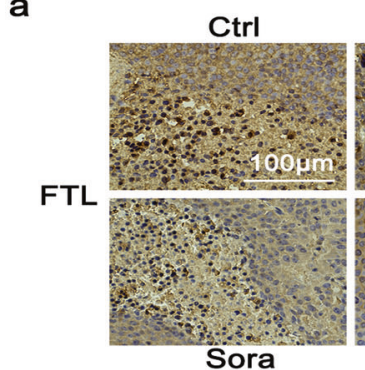

b

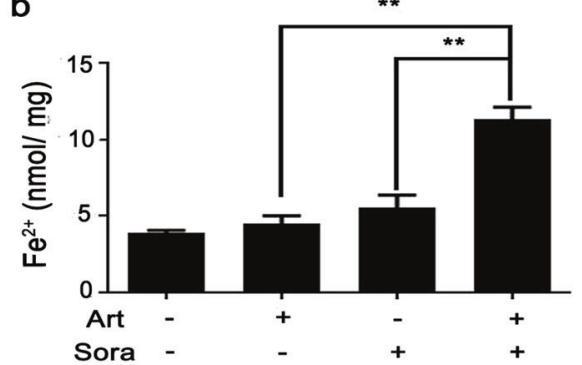

Art
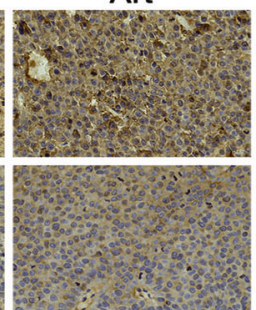

$A+S$

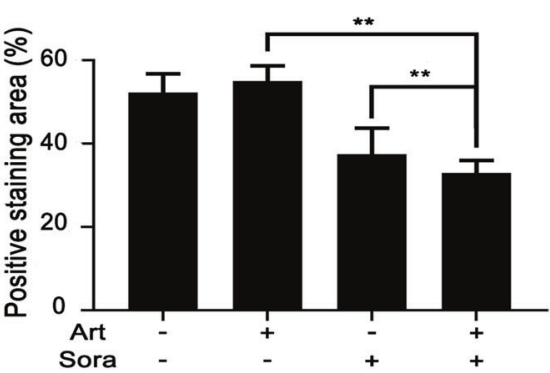

C

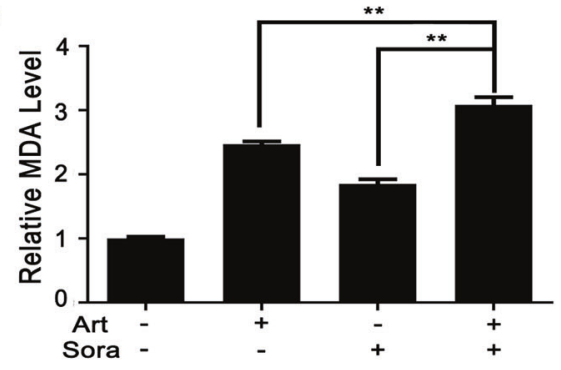

d

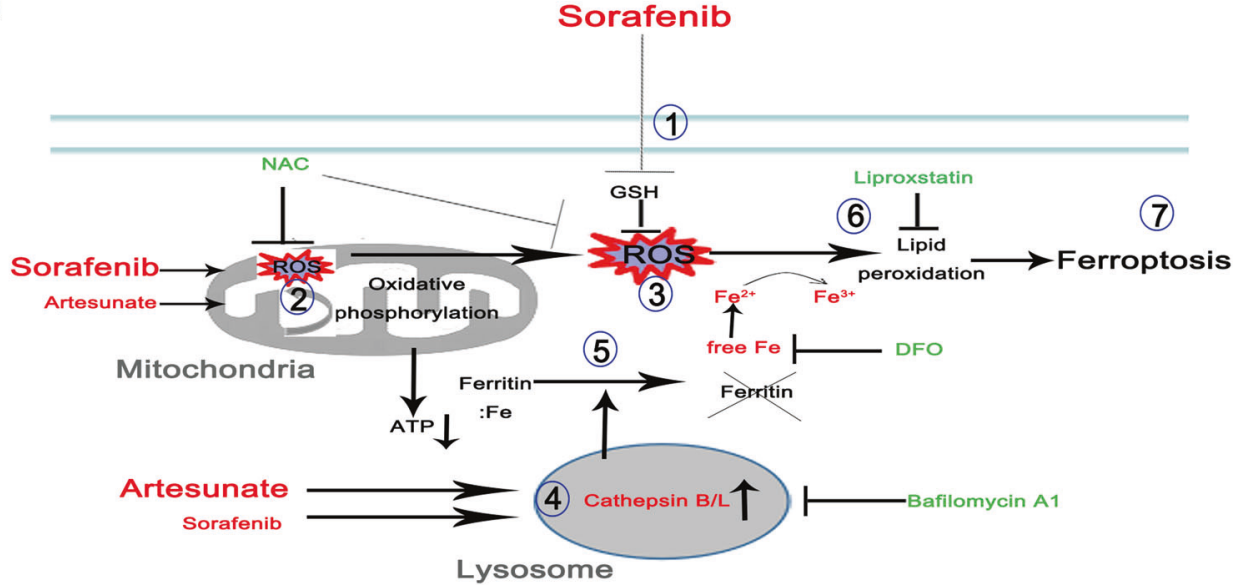

Fig. 6 The combined treatments caused consistent changes in FTL degradation and MDA production in xenograft nodules in vivo. a Xenograft nodules were collected as shown in Fig. 2b. FTL protein expression was determined by IHC staining as shown in Fig. $2 \mathrm{~d}$. The contents of free $\mathrm{Fe}^{2+}$ ions (b) and MDA (c) in tumor nodules were quantified with respective biochemical assay kits. ${ }^{* *} P<0.01$. $\mathbf{d}$ The cartoon describes the molecular mechanisms through which the combined treatment led to ferroptosis. Sorafenib directly inhibits (1) GSH synthesis and (2) mitochondrial functions, which contributes to (3) extensive oxidative stress. Artesunate primarily (4) promotes lysosomal activation and then synergizes with sorafenib to activate cathepsin B/L activities followed by (5) ferritin degradation. The convergence of sorafenib-induced oxidative stress and artesunate-mediated ferritinophagy causes (6) lipid peroxidation and eventually (7) ferroptosis. DFO, deferoxamine mesylate; GSH glutathione, NAC N-acetyl cysteine; ROS reactive oxygen species. The red color indicates ferroptosis inducers or essential aspects of ferroptosis. The green color indicates inhibitors of ferroptosis induction.

sorafenib sensitivity, while the induction of serum metallothionein-1 after sorafenib treatment indicates poorer overall survival in HCC patients [38]. Second, some HCC patients are intolerant to sorafenib-induced side effects. Artesunate, which is clinically available, could be an ideal sensitizing agent for increasing the effects of sorafenib-resistance HCC at a dose below the toxic threshold. However, the validity of these proposed effects requires further clinical investigations. The current preclinical study was conducted in an ectopic xenograft tumor model inoculated with HCC cells. Primary rodent HCC models and patient-derived xenograft (PDX) models may better mimic the pathology observed in the clinic. These models should be further explored in future studies.

\section{CONCLUSIONS}

In conclusion, the present study demonstrated that artesunate and low-dose sorafenib have a synergistic effect in inducing HCC ferroptosis in vitro and in vivo. Their distinct mechanisms involved inducing oxidative stress and lysosomal activation work together to induce ferroptosis. Because artesunate is well tolerated and affordable, its combination with sorafenib may benefit a large proportion of HCC patients. Furthermore, this combined treatment may decrease potential unwanted sorafenib toxicity by lowering its effective dosage.

\section{ACKNOWLEDGEMENTS}

This work was supported by the National Natural Science Foundation of China (81672370 and 81972291), the Guangxi Natural Science Foundation Key Grant (2018GXNSFDA050006), the Hundred-Talent Program (2016), the Guangxi Medical University Training Program for Distinguished Young Scholars (2017), the OpenGrant from Key Laboratory of High-Incidence Tumor Prevention \& Treatment (Guangxi Medical University, GKE2018-02) (all awarded to GDL); the Guangxi Natural Science Foundation Key Grant (2017GXNSFDA198020) awarded to JZ, and the Guangxi Specially Appointed Professor Grant awarded to JW. 


\section{AUTHOR CONTRIBUTIONS}

$\mathrm{ZJL}, \mathrm{XWH}$, and HQD equally performed the majority of experiments and analyzed the data; $Z J L, X W H$, and YJL performed the nude mouse xenograft experiment; HQD and JF conducted the Seahorse metabolic experiments and flow cytometry analysis; YW prepared the figures; XMY, ZXW, and JHD provided technical support; PHC and HS performed the literature review; JGW and GDL designed the research and summarized the data; JZ and GDL wrote the paper.

\section{ADDITIONAL INFORMATION}

The online version of this article (https://doi.org/10.1038/s41401-020-0478-3) contains supplementary material, which is available to authorized users.

Competing interests: The authors declare no competing interests.

\section{REFERENCES}

1. Villanueva A, Hernandez-Gea V, Llovet JM. Medical therapies for hepatocellular carcinoma: a critical view of the evidence. Nat Rev Gastroenterol Hepatol. 2012;10:34-42.

2. European Association for the Study of the Liver. EASL clinical practice guidelines: management of hepatocellular carcinoma. J Hepatol. 2018;69:182-236.

3. Llovet JM, Ricci S, Mazzaferro V, Hilgard P, Gane E, Blanc JF, et al. Sorafenib in advanced hepatocellular carcinoma. N Engl J Med. 2008;359:378-90.

4. Cheng AL, Kang YK, Chen Z, Tsao CJ, Qin S, Kim JS, et al. Efficacy and safety of sorafenib in patients in the Asia-Pacific region with advanced hepatocellular carcinoma: a phase III randomised, double-blind, placebo-controlled trial. Lancet Oncol. 2009;10:25-34.

5. Llovet JM, Montal R, Sia D, Finn RS. Molecular therapies and precision medicine for hepatocellular carcinoma. Nat Rev Clin Oncol. 2018;15:599-616.

6. Yau T, Yao TJ, Chan P, Wong H, Pang R, Fan ST, et al. The significance of early alpha-fetoprotein level changes in predicting clinical and survival benefits in advanced hepatocellular carcinoma patients receiving sorafenib. Oncologist. 2011;16:1270-9.

7. Faivre S, Rimassa L, Finn RS. Molecular therapies for HCC: looking outside the box. J Hepatol. 2020;72:342-52.

8. Newell P, Toffanin S, Villanueva A, Chiang DY, Minguez B, Cabellos L, et al. Ras pathway activation in hepatocellular carcinoma and anti-tumoral effect of combined sorafenib and rapamycin in vivo. J Hepatol. 2009;51:725-33.

9. Jung DH, Tak E, Hwang S, Song GW, Ahn CS, Kim KH, et al. Antitumor effect of sorafenib and mTOR inhibitor in liver transplantation recipients with hepatocellular carcinoma recurrence. Liver Transpl. 2018;24:932-45.

10. Lin $\mathrm{CH}$, Elkholy $\mathrm{KH}$, Wani NA, Li D, Hu P, Barajas JM, et al. Ibrutinib potentiates anti-hepatocarcinogenic efficacy of sorafenib by targeting EGFR in tumor cells and BTK in immune cells in the stroma. Mol Cancer Ther. 2020;19:384-96.

11. Ou DL, Shen YC, Liang JD, Liou JY, Yu SL, Fan HH, et al. Induction of Bim expression contributes to the antitumor synergy between sorafenib and mitogen-activated protein kinase/extracellular signal-regulated kinase kinase inhibitor $\mathrm{Cl}-1040$ in hepatocellular carcinoma. Clin Cancer Res. 2009;15:5820-8.

12. Chen KF, Yu HC, Liu TH, Lee SS, Chen PJ, Cheng AL. Synergistic interactions between sorafenib and bortezomib in hepatocellular carcinoma involve PP2Adependent Akt inactivation. J Hepatol. 2010;52:88-95.

13. Zhu AX, Kang YK, Yen CJ, Finn RS, Galle PR, Llovet JM, et al. Ramucirumab after sorafenib in patients with advanced hepatocellular carcinoma and increased afetoprotein concentrations (REACH-2): a randomised, double-blind, placebocontrolled, phase 3 trial. Lancet Oncol. 2019;20:282-96.

14. Wei G, Wang M, Hyslop T, Wang Z, Carr BI. Vitamin K enhancement of sorafenibmediated HCC cell growth inhibition in vitro and in vivo. Int J Cancer. 2010;127:2949-58.

15. Pal HC, Baxter RD, Hunt KM, Agarwal J, Elmets CA, Athar M, et al. Fisetin, a phytochemical, potentiates sorafenib-induced apoptosis and abrogates tumor growth in athymic nude mice implanted with BRAF-mutated melanoma cells. Oncotarget. 2015;6:28296-311.

16. Chen JH, Chiang IT, Hsu FT. Protein kinase B inactivation is associated with magnolol-enhanced therapeutic efficacy of sorafenib in hepatocellular carcinoma in vitro and in vivo. Cancers. 2019;12:87. https://doi.org/10.3390/cancers12010087
17. Wu WD, Chen PS, Omar HA, Arafa ES, Pan HW, Jeng J, et al. Antrodia cinnamomea boosts the anti-tumor activity of sorafenib in xenograft models of human hepatocellular carcinoma. Sci Rep. 2018;8:12914. https://doi.org/10.1038/s41598018-31209-8

18. Dixon SJ, Lemberg KM, Lamprecht MR, Skouta R, Zaitsev EM, Gleason CE, et al. Ferroptosis: an iron-dependent form of non-apoptotic cell death. Cell. 2012;149:1060-72.

19. Stockwell BR, Angeli FJP, Bayir H, Bush Al, Conrad M, et al. Ferroptosis: aregulated cell death nexus linking metabolism, redox biology, and disease. Cell. 2017;171:273-85.

20. Dixon SJ, Patel DN, Welsch M, Skouta R, Lee ED, Hayano M, et al. Pharmacological inhibition of cystine-glutamate exchange induces endoplasmic reticulum stress and ferroptosis. eLife. 2014;3:e02523. https://doi.org/10.7554/eLife.02523

21. Eling N, Reuter L, Hazin J, Hamacher-Brady A, Brady NR. Identification of artesunate as a specific activator of ferroptosis in pancreatic cancer cells. Oncoscience. 2015;2:517-32.

22. Sun $X$, Yan $P$, Zou $C$, Wong $Y K$, Shu $Y$, Lee $Y M$, et al. Targeting autophagy enhances the anticancer effect of artemisinin and its derivatives. Med Res Rev. 2019;39:2172-93.

23. Wang J, Xu C, Wong YK, Li Y, Liao F, Jiang T, et al. Artemisinin, the magic drug discovered from traditional chinese medicine. Engineering. 2018;5:32-9.

24. Wang J, Zhang CJ, Chia WN, Loh CCY, Li Z, Lee YM, et al. Haem-activated promiscuous targeting of artemisinin in plasmodium falciparum. Nat Commun. 2015;6:10111. https://doi.org/10.1038/ncomms10111

25. Yang ND, Tan SH, Ng S, Shi Y, Zhou J, Tan KSW, et al. Artesunate induces cell death in human cancer cells via enhancing lysosomal function and lysosomal degradation of ferritin. J Biol Chem. 2014;289:33425-41.

26. Chou TC. Drug combination studies and their synergy quantification using the Chou-Talalay method. Cancer Res. 2010;70:440-6.

27. Zhou J, Tan SH, Nicolas V, Chantal B, Yang ND, Zhang J, et al. Activation of lysosomal function in the course of autophagy via MTORC1 suppression and autophagosome-lysosome fusion. Cell Res. 2013;23:508-23.

28. Schneider CA, Rasband WS, Eliceiri KW. NIH Image to ImageJ: 25 years of image analysis. Nat Methods. 2012;9:671-5.

29. Lu GD, Ang YH, Zhou J, Tamilarasi J, Yan B, Lim YC, et al. CCAAT/enhancer binding protein a predicts poorer prognosis and prevents energy starvation-induced cell death in hepatocellular carcinoma. Hepatology. 2015;61:965-78.

30. Roh JL, Kim EH, Jang H, Shin D. Nrf2 inhibition reverses the resistance of cisplatinresistant head and neck cancer cells to artesunate-induced ferroptosis. Redox Biol. 2017;11:254-62.

31. Greenshields AL, Shepherd TG, Hoskin DW. Contribution of reactive oxygen species to ovarian cancer cell growth arrest and killing by the anti-malarial drug artesunate. Mol Carcinog. 2017;56:75-93.

32. Nakase I, Gallis B, Takatani-Nakase T, Oh S, Lacoste E, Singh NP, et al. Transferrin receptor-dependent cytotoxicity of artemisinin-transferrin conjugates on prostate cancer cells and induction of apoptosis. Cancer Lett. 2009;274:290-8.

33. Jeong DE, Song HJ, Lim S, Lee SJ, Lim JE, Nam DH, et al. Repurposing the antimalarial drug artesunate as a novel therapeutic agent for metastatic renal cell carcinoma due to its attenuation of tumor growth, metastasis, and angiogenesis. Oncotarget. 2015;6:33046-64.

34. Vandewynckel YP, Laukens D, Geerts A, Vanhove C, Descamps B, Colle I, et al. Therapeutic effects of artesunate in hepatocellular carcinoma: repurposing an ancient antimalarial agent. Eur J Gastroenterol Hepatol. 2014;26:861-70.

35. Wu J, Liu S, Xing YR, Zhao RP, Xie J, Hu D, et al. Artesunate promotes sensitivity to sorafenib in hepatocellular carcinoma. Biochem Biophys Res Commun. 2019:519:41-5.

36. Doll S, Proneth B, Tyurina YY, Panzilius E, Kobayashi S, Ingold I, et al. ACSL4 dictates ferroptosis sensitivity by shaping cellular lipid composition. Nat Chem Biol. 2017;13:91-8.

37. Feng J, Lu PZ, Zhu GZ, Hooi SC, Wu Y, Huang XW, et al. ACSL4 is a predictive biomarker of sorafenib sensitivity in hepatocellular carcinoma. Acta Pharmacol Sin. 2020. https://doi.org/10.1038/s41401-020-0439-x.

38. Houessinon A, François C, Sauzay C, Louandre C, Mongelard G, Godin C, et al. Metallothionein-1 as a biomarker of altered redox metabolism in hepatocellular carcinoma cells exposed to sorafenib. Mol Cancer. 2016;15:38. https://doi.org/ 10.1186/s12943-016-0526-2 